\title{
The Effect of Surgical Intervention on Pediatric Burn Injury Survival in a Resource-Poor Setting
}

\author{
Laura N. Purcell, MD, MPH, ${ }^{a}$ Wone Banda, MB, BS, ${ }^{b}$ \\ Brittney Williams, MD, ${ }^{a}$ Jared Gallaher, MD, $\mathrm{MPH},{ }^{a}$ \\ and Anthony Charles, MD, MPH ${ }^{a, b, *}$
}

${ }^{a}$ Department of Surgery, University of North Carolina at Chapel Hill, Chapel Hill, North Carolina

${ }^{\mathrm{b}}$ Department of Surgery, Kamuzu Central Hospital, Lilongwe, Malawi

\section{A R T I C L E I N F O}

Article history:

Received 23 November 2019

Received in revised form

18 March 2020

Accepted 24 March 2020

Available online 23 April 2020

Keywords:

Burn injury

Burn outcomes in resource poor settings

Surgical intervention and burn mortality
A B S T R A C T

Introduction: Burns are one of the most common injuries sustained globally. Low- and middle-income countries (LMICs) are disproportionately affected by burn injury morbidity and mortality; African children have the highest burn mortality globally. In high-income countries, early surgical intervention has shown to improve survival. However, when applied to burn victims in LMICs, improved survival in the early excision cohort $(\leq 5 \mathrm{~d})$ was not seen. Therefore, we aimed to determine the magnitude of the effect of surgical intervention on burn injury survival.

Methods: A retrospective analysis of a prospectively collected data, utilizing the Kamuzu Central Hospital Burn Database from May 2011 to July 2019, was performed. Pediatric patients ( $\leq 12$ y) were included. Patients were excluded if they underwent surgical intervention for nonacute burn care management. Bivariate analyses stratifying by type of surgical intervention was performed, comparing demographics, burn characteristics, surgical intervention, and patient mortality. Standardized estimates were adjusted using the inverse-probability of treatment weights to account for confounding. Weighted logistic regression modeling was performed to determine the odds of mortality based on if a patient underwent surgical intervention.

Results: During the study, 2364 patients were seen at the Kamuzu Central Hospital, 1785 (75.5\%) were children $\leq 12$ y who met inclusion criteria. In the overall cohort, 342 (19.2\%) underwent operations, including split-thickness skin graft $(n=196,57.3 \%)$, debridement ( $n=116,33.9 \%)$, escharotomy $(n=19,5.6 \%)$, and amputation $(n=1,0.3 \%)$. The surgery cohort was older $(4.2 \pm 3.1$ versus $3.1 \pm 2.6 \mathrm{y}, \mathrm{P}<0.001)$ with larger percent total body surface area burns (16\%, interquartile range: $10-24$ versus $13 \%$, interquartile range: $8-20$, $P<0.001)$ than those who did not have surgery. In the propensity score-weighted logistic regression predicting survival, patients undergoing surgery after burn injury had an increased odds of survival (odds ratio: $5.24,95 \%$ confidence interval: $2.40-11.44, P=0.003$ ) when compared with patients not undergoing surgery.

\footnotetext{
All persons who meet authorship criteria are listed as authors, and all authors certify that they have participated sufficiently in the work to take public responsibility for the content, including participation in the concept, design, analysis, writing, or revision of the manuscript. Furthermore, each author certifies that this material or similar material has not been and will not be submitted to or published in any other publication before its appearance.

Funding: This study was supported in part by the NIH Fogarty International Center Postdoctoral Research Fellowship to Dr. Purcell.

* Corresponding author. UNC School of Medicine, 4008 Burnett Womack Building CB, Chapel Hill, 7228 NC. Tel.: 9199664388 ; fax: 9199660369.

E-mail address: anthchar@med.unc.edu (A. Charles).
} 
Conclusions: In this propensity-weighted analysis, surgical intervention following burn injury increases the odds of survival by a factor of 5.24 when compared with patients not undergoing surgical intervention. Efforts to enhance burn infrastructure to deliver surgical care is imperative to attenuate burn mortality in resource-poor settings.

\section{Introduction}

Burn injury is the fourth most common type of trauma worldwide, after traffic injuries, falls, and interpersonal violence. ${ }^{1}$ Burn injury disproportionally affects people in lowand middle-income countries (LMICs). ${ }^{2}$ Mortality from pediatric burns is most pronounced in LMICs, where over $90 \%$ of burn-related pediatric deaths occur. ${ }^{3}$ This equates to 3.4 burnrelated deaths per 100,000 children in LMICs compared with 0.5 deaths per 100,000 children in high-income countries. ${ }^{4}$ The disparity is particularly prominent in Africa, which has the highest pediatric burn mortality in the world. ${ }^{5}$

Pediatric burn mortality is usually attributable to burn shock in the first hours after injury, respiratory failure in the following days, and septic complications and organ failure during the subsequent weeks. ${ }^{6}$ In resource-poor settings, burn care is complicated by numerous factors such as delays in patient presentation, financial constraints, and poor health system infrastructure, including a paucity of specialty-trained health care personnel, scarcity of well-developed burn units, and insufficient access to the operating room. Furthermore, the lack of standardized burn care protocols has a detrimental impact on burn care and outcomes in sub-Saharan Africa.

After effective fluid resuscitation and stabilization of the critically ill burn-injured child, attention is directed toward burn wound management. The key elements of nonoperative burn wound management include cleansing, topical antimicrobial agents, and dressing changes. ${ }^{7}$ Definitive surgical management of the wound in a resource-poor setting is usually limited to debridement, excision, and split-thickness skin grafting. Burned children in resource-poor settings are primarily managed nonoperatively. ${ }^{8-10}$

We, therefore, aimed to evaluate the effect of surgical intervention following acute burn injury in a pediatric cohort treated at a dedicated burn unit in Malawi. Specifically, we were interested in the magnitude of the effect of surgical intervention on burn injury survival.

\section{Methods}

A retrospective review of prospectively collected data was performed utilizing the Kamuzu Central Hospital (KCH) Burn Registry from May 2011 until July 2019. All patients who are admitted to the $\mathrm{KCH}$ burn unit are included in the registry. $\mathrm{KCH}$, a public hospital primarily supported by the Malawi Ministry of Health, is a 900-bed tertiary hospital located in Lilongwe, Malawi. It serves the capital city and the 6 million persons who live in central Malawi. The KCH burn unit is a 31bed unit, staffed by a consultant Malawian plastic surgeon, two specialized Malawian burn clinical officers, and a burntrained nursing staff. Visiting surgeons from partner institutions in high-income countries were not involved in the surgical care of burn injured patients during the time of the study.

Inclusion criteria for this study were pediatric patients $\leq 12 \mathrm{y}$ who were admitted and underwent burn operations in the acute period, including wound debridement, splitthickness skin grafts, escharotomies and fasciotomies, and amputations. Decision to undergo surgical care was determined clinically by the surgeon and clinical officers. As early excision and grafting has not proven to have a survival benefit in our pediatric population, the general approach of the burn unit is to delay operative intervention, specifically splitthickness skin graft until postburn day $5 .{ }^{11}$ Patients were excluded if they were adults or undergoing operations for function or cosmesis, including contracture release.

Univariate analysis was performed to evaluate missing data and data distribution. Surgery was collapsed into groups that did or did not receive an operative intervention. All types of operative intervention were not able to be meaningfully analyzed independently because of cohort size, specifically amputations and escharotomies. Bivariate analysis was completed, stratifying over whether a patient underwent a surgical intervention during hospital admission. The central tendency of normally distributed covariates was described with means and standard deviations, whereas non-normally distributed covariates were reported with medians and interquartile ranges (IQRs). During bivariate analysis, $\chi^{2}$ for categorical variables, Student's t-test for normally distributed continuous variables, and Kruskal-Wallis for not normally distributed continuous variables were used to compare exposure distribution.

A propensity score analysis was performed to reduce bias of patient and injury characteristics to better determine survival based on surgical intervention. The propensity score was performed with a logistic regression using surgery as the dependent variable. Independent variables included age, sex, time to presentation, percent total body surface area of burn (\%TBSA), mechanism of burn injury, prehospital treatment by a traditional healer, and receiving a transfusion or antibiotics. The propensity score was inversed to calculate the inverse probability of treatment weight, to weight the groups based on surgical intervention. After weighting, we confirmed balance as there was no statistical difference between the cohorts who did or did not undergo operative intervention in the logistic regression as previously described $(P>0.05)$.

To determine the influence of the operative intervention on survival after burn injury, we performed a propensity score-weighted logistic regression. Covariates included a priori in the logistic regression model were age, sex, time to presentation, \%TBSA, mechanism of burn, and preadmission treatment by a traditional health practitioner. On bivariate analysis, treatment with antibiotics and receiving a 
Table 1 - Unadjusted demographics by surgery.

\begin{tabular}{|c|c|c|c|c|}
\hline Variables & Overall $n=1785$ & No surgery $n=1443$ & Surgery $n=342$ & $P$ value \\
\hline Age: $\mu$ (SD) & $3.3(2.7)$ & $3.1(2.6)$ & $4.2(3.1)$ & $<0.001$ \\
\hline Male sex: $n(\%)$ & $993(55.8)$ & $823(57.2)$ & $170(49.9)$ & 0.01 \\
\hline Burn total body surface area: Median (IQR) & $14(8-21)$ & $13(8-20)$ & $16(10-24)$ & $<0.001$ \\
\hline Mechanism of burn: $n(\%)$ & & & & $<0.001$ \\
\hline Scald-Water & $986(55.5)$ & $896(62.3)$ & $90(26.5)$ & \\
\hline Scald-Grease & $290(16.3)$ & $244(17.0)$ & $46(13.5)$ & \\
\hline Flame & $484(27.2)$ & $282(19.6)$ & $202(59.4)$ & \\
\hline Contact & $6(0.3)$ & $5(0.4)$ & $1(0.3)$ & \\
\hline Electrical/Lightning & $12(0.7)$ & $11(0.8)$ & $1(0.3)$ & \\
\hline Traditional medicine used: $n(\%)$ & $185(10.9)$ & $142(10.3)$ & $43(13.2)$ & 0.1 \\
\hline Time to presentation: $n(\%)$ & & & & $<0.001$ \\
\hline $0-6 \mathrm{~h}$ & 209 (11.9) & $168(11.9)$ & $41(12.2)$ & \\
\hline $7-12 \mathrm{~h}$ & $392(22.4)$ & $332(23.5)$ & $71(21.1)$ & \\
\hline $12-24 \mathrm{~h}$ & $628(35.8)$ & $557(39.4)$ & $71(21.1)$ & \\
\hline $24-48 \mathrm{~h}$ & $67(3.9)$ & $54(3.8)$ & $13(3.9)$ & \\
\hline$>48 \mathrm{~h}$ & $456(26.0)$ & $304(21.5)$ & $152(45.1)$ & \\
\hline Antibiotics prescribed: $n(\%)$ & $938(53.2)$ & $718(50.4)$ & $220(65.3)$ & $<0.001$ \\
\hline Transfused: $n(\%)$ & $242(43.8)$ & $85(23.6)$ & $157(81.4)$ & $<0.001$ \\
\hline Mean upper arm circumference: $n(\%)$ & & & & 0.6 \\
\hline Severe acute malnutrition & $6(0.9)$ & $5(0.9)$ & $1(0.6)$ & \\
\hline Moderate acute malnutrition & $8(1.2)$ & $6(1.1)$ & $2(1.3)$ & \\
\hline Risk for acute malnutrition & $50(7.2)$ & $35(6.5)$ & $15(9.6)$ & \\
\hline Well-nourished & $631(90.8)$ & $492(91.5)$ & $139(88.5)$ & \\
\hline Hospital length of stay: Median (IQR) & $11(6-24)$ & $9(5-16)$ & $39(24-61)$ & $<0.001$ \\
\hline Mortality: $n(\%)$ & $279(15.9)$ & $244(17.2)$ & 35 (10.5) & 0.002 \\
\hline
\end{tabular}

transfusion were statistically significant and included in the model. A backward elimination approach was performed to reduce error in the model with the removal of covariates from the model based on $P$ value $(P<0.05)$. Based on this criterion, treatment with antibiotics and sex were removed from the final model as they were not statistically significant in the multiple logistic regression. There was a reduction of bias and improved precision as there was a narrowing of confidence intervals (CIs) and $<10 \%$ change seen in coefficients, respectively. A receiver operating characteristic analysis, to calculate a c-statistic, Akaike information criterion (AIC), and Bayesian information criterion (BIC) were performed for the final and full model to determine the model fit.

This analysis was performed using StataCorp v16.0, College Station, TX. CIs are reported at 95\%, and alpha was set at 0.05 for this study. An approved waiver for informed consent was obtained. The Malawi National Health Science Research Committee and the University of North Carolina Institutional Review Boards approved this study.

\section{Results}

From May 2011 to August 2019, 2364 patients were captured in the KCH Burn Registry, which included 1794 (75.9\%) children $\leq 12$ y. Of the children, 1785 (99.5\%) met inclusion criteria. In the overall cohort, 342 (19.2\%) underwent operations, including split-thickness skin grafting ( $n=196,57.3 \%)$, debridement $(n=116,33.9 \%)$, escharotomy $(n=19,5.6 \%)$, and amputation ( $n=1,0.3 \%)$. The median time to operation was $16 \mathrm{~d}$ (IQR 7-32). The overall cohort had a median age of $3.0 \mathrm{y}$ (IQR: $1-4$ y) was predominately male $(n=993,55.8 \%)$, with a median \%TBSA of $14 \%$ (IQR: $8 \%-21 \%$ ). The surgery cohort was older (4.2 \pm 3.1 versus $3.1 \pm 2.6 \mathrm{y}, \mathrm{P}<0.001)$ with larger \%TBSA burns (16\%, IQR: $10-24$ versus $13 \%$, IQR: $8-20, P<0.001)$ than those who did not have surgery. The primary mechanism of burn in the surgical cohort was flame $(n=202,59.4 \%)$ versus scald ( $n=896,62.3 \%$ ) in the no surgery cohort $(P<0.001)$. A higher proportion of patients presented later than $48 \mathrm{~h}$ after burn in the surgical cohort compared with the nonsurgical cohort who had the highest proportion $(n=557,39.4 \%)$ present $12-24 \mathrm{~h}$ after burn, $(P<0.001)$. The overall mortality in the cohort was 279 (15.9\%), with a mortality of 35 (10.5\%) and 244 (17.2 \%) from the surgical and nonsurgical cohorts, respectively $(P<0.001)$, Table 1 . Following bivariate analysis, we utilized propensity scores to calculate inverse propability treatment weights and after weighting we confirmed balance (Table 2).

The final model's receiver operating characteristic curve had a c-statistic of 0.806 . There was no difference in Akaike 
Table 2 - Logistic regression predicting surgery before and after propensity score weighted matching.

\begin{tabular}{|c|c|c|c|c|c|c|}
\hline \multirow[t]{2}{*}{ Variables } & \multicolumn{3}{|c|}{ Unweighted logistic regression } & \multicolumn{3}{|c|}{ Weighted logistic regression } \\
\hline & Odds ratio & $95 \%$ CI & $P$ value & Odds ratio & $95 \%$ CI & $P$ value \\
\hline Age & 1.08 & $0.98-1.19$ & 0.1 & 1.03 & $0.93-1.15$ & 0.6 \\
\hline Sex & 0.97 & $0.56-1.66$ & 0.9 & 1.06 & $0.58-1.95$ & 0.9 \\
\hline \multicolumn{7}{|l|}{ Time to presentation } \\
\hline $0-6 \mathrm{~h}$ & Ref & ${ }^{*}$ & * & Ref & * & $\cdot$ \\
\hline $7-12 \mathrm{~h}$ & 2.15 & $0.95-4.86$ & 0.07 & 0.94 & $0.40-2.21$ & 0.9 \\
\hline $12-24 \mathrm{~h}$ & 2.65 & $1.09-6.42$ & 0.03 & 0.89 & $0.36-2.20$ & 0.8 \\
\hline $24-48 \mathrm{~h}$ & 5.44 & $1.35-21.96$ & 0.02 & 0.95 & $0.21-4.25$ & 1.0 \\
\hline$>48 \mathrm{~h}$ & 2.51 & $1.20-5.28$ & 0.02 & 0.83 & $0.37-1.90$ & 0.7 \\
\hline Percent TBSA & 0.96 & $0.94-0.98$ & 0.001 & 1.02 & $0.99-1.05$ & 0.2 \\
\hline \multicolumn{7}{|l|}{ Mechanism } \\
\hline Scald-Water & Ref & * & ${ }^{*}$ & Ref & - & $\cdot$ \\
\hline Scald-Grease & 1.30 & $0.59-2.84$ & 0.5 & 0.81 & $0.34-1.93$ & 0.6 \\
\hline Flame & 3.57 & $1.89-6.72$ & $<0.001$ & 0.78 & $0.41-1.50$ & 0.5 \\
\hline Traditional medicine & 0.75 & $0.38-1.50$ & 0.4 & 1.94 & $0.89-4.20$ & 0.09 \\
\hline Antibiotics prescribed & 0.72 & $7.27-25.19$ & 0.3 & 1.26 & $0.65-2.43$ & 0.5 \\
\hline Transfused & 13.53 & $0.04-0.25$ & $<0.001$ & 0.97 & $0.52-1.80$ & 0.9 \\
\hline
\end{tabular}

" $P$ value $<0.05$.

information criterion between the full (0.827) and final models (0.831), as the difference was less than two. The BIC was calculated to be -1930.5 and -1979.7 in the full and final model, respectively. The BIC absolute difference between the two models was 49.2 , indicating there is very strong evidence toward using the final compared with the full model.

In the final propensity score-weighted logistic regression model predicting survival, undergoing surgery after burn injury had an increased odds of survival (odds ratio [OR] 5.24, 95\% CI 2.40-11.44, P < 0.001) when compared to patients not undergoing surgery. Increasing \%TBSA (OR: 0.94, 95\% CI: 0.91-0.97, $P<0.001$ ) and receiving a transfusion (OR 0.30, 95\% CI 0.12-0.76, $P=0.01$ ) had lower odds of survival, Table 3.

\section{Discussion}

Surgical intervention remains the cornerstone of burn wound management. In this study, we show surgical intervention following burn injury increases the odds of burn injury survival by a factor of 5.24 when compared with patients not undergoing surgical intervention, after controlling for pertinent covariates in a propensity weighted analysis.

In developed countries, surgical management such as early excision and skin grafting leads to decreased hospital lengths of stay, a reduced cost of hospital care, and a significant reduction in mortality. ${ }^{12-15}$ During early, aggressive surgical debridement, nonviable tissue is removed. As the nidus for infection is removed, the wound bed is less likely to become infected. Further, the removal of necrotic tissue has the potential to reduce the production of chemical mediators which stimulate the inflammatory cascade leading to multisystem organ failure. $^{16}$
However, we have previously shown early operative intervention (before postburn day 5) increases mortality in the pediatric burn cohort in Malawi. ${ }^{11}$ In addition to an increased

Table 3 - Weighted logistic regression predicting survival.

\begin{tabular}{lccc} 
Variables & \multicolumn{3}{c}{ Weighted logistic regression } \\
\cline { 2 - 4 } & Odds ratio & $95 \%$ CI & P value \\
\hline Surgery & 5.24 & $2.40-11.44$ & $<0.001$ \\
\hline Age & 1.09 & $0.93-1.26$ & 0.3 \\
\hline Percent TBSA & 0.94 & $0.91-0.97$ & $<0.001$ \\
\hline Time to presentation & & & \\
$\quad 0-6 \mathrm{~h}$ & Ref & & \\
\hline 7-12 h & 0.59 & $0.19-1.89$ & 0.4 \\
\hline 12-24 h & 0.68 & $0.18-2.56$ & 0.6 \\
\hline 24-48 h & 0.69 & $0.10-4.66$ & 0.7 \\
\hline >48 h & 0.51 & $0.16-1.66$ & 0.3 \\
\hline Mechanism & & & \\
\hline Scald-Water & Ref & & \\
\hline Scald-Grease & 0.43 & $0.17-1.13$ & 0.09 \\
\hline Flame & 0.39 & $0.15-1.01$ & 0.05 \\
\hline Transfused & 0.30 & $0.12-0.76$ & 0.01
\end{tabular}

C-statistic: $0.806, P=0.7$.

Akaike Information Criterion.

Full Model: 0.827.

Final Model: 0.831 .

Bayesian Information Criterion.

Full Model: -1930.5.

Final Model: -1979.7.

Difference: 49.2.

${ }^{*} \mathrm{P}$ value $<0.05$. 
risk of mortality, early excision and grafting is not routinely employed in resource-poor settings for several reasons. ${ }^{17}$ In most centers, access to the operating room is limited because of resource and staffing limitations. Operative intervention for burn patients may be postponed as their surgical care is not regarded as emergent compared with other patients with acute care general surgery conditions, or preexisting malnutrition may be prohibitive to optimal wound healing. ${ }^{18}$ There are also increased blood transfusion requirements following tangential wound excision performed in burn surgery. ${ }^{19}$ With limited availability of blood and blood products, surgical intervention for burns may be regarded as prohibitive. ${ }^{20}$

The overall mortality within the pediatric burn cohort in this study was $15.9 \%$. This is comparable to mortality from other burn centers in the region. Hyder et al. reported a casefatality of $6-10 \%$ and between 18,000 and 30,000 burn deaths annually among African children. ${ }^{21}$ This is contrasted by reports from Cameron, which reveal pediatric burn mortality rates of up to $41 \%{ }^{22}$ These mortality rates indicate the need for increasing surgical intervention to further attenuate mortality.

There is a paucity of data on the use of traditional medicine in LMICs. ${ }^{23,24}$ Traditional medicine is commonly used among our patient population in Malawi because of the proximity of traditional medicine providers compared with local health centers and district hospitals. In nearby Mozambique, nearly two-thirds of guardians take pediatric patients to traditional healers before reaching a health-care center. ${ }^{25}$ Some traditional medicine therapies commonly used in Malawi include cattle manure, papaya leaves, tomato leaves, honey, raw eggs, and aloe vera.

The surest way to reduce burn mortality is a comprehensive burn prevention strategy. Education for parents and children about the burn risks with hot objects and fluid, open fires, as well as the safe storage of flammable and toxic substances is needed. ${ }^{26}$ This includes a robust education program on preventing cooking-related fires, safety surrounding the use of paraffin lamps for heating, and adequate oversight of children within the family unit. Important burn prevention interventions that can be implemented are to raise cooking fires on bricks or stones and guard of open fires from children by using fire grids. ${ }^{27}$

Most governments and public health initiatives in subSaharan Africa have failed to invest in surgical infrastructure as a whole. There is a persistent emphasis from wellfunded foreign aid initiatives on the prevention and treatment of malnutrition, obstetric disorders, and communicable diseases, such as HIV, malaria, and tuberculosis. This has perpetuated the misperception that surgical intervention is not a cost-effective endeavor and has shaped local health-care policy to the detriment of patients with surgical diseases. A deliberate policy of investing in the surgical ecosystem, increasing the surgical workforce, allocating space for the delivery of surgical care, and providing the necessary supplies to achieve surgical care is imperative. ${ }^{28}$ Importantly, burn care is an integral part of essential surgical care.

This study is limited by its retrospective methodology and that the data are from a single-burn center. Furthermore, the surgical intervention we considered was not limited to early excision and grafting but included escharotomy, amputation, and debridement. In addition, we did not differentiate between early versus late surgical intervention. However, in this propensity-weighted analysis, we balanced the surgical and nonsurgical cohorts for all well-established predictors of mortality, specifically age and percent total body surface area burns.

\section{Conclusions}

In this study, we show that surgical intervention in pediatric burn patients may result in a survival benefit. Attenuating burn mortality on resource-poor settings is still dependent on primary burn prevention. Increased efforts to enhance the delivery of operative burn care in resource-poor settings are imperative.

\section{Acknowledgment}

Author contribution: Laura Purcell contributed to drafting of manuscript. Wone Banda contributed to acquisition of data and manuscript revision. Brittney Williams contributed to drafting of manuscript and manuscript revision. Jared Gallaher contributed to acquisition of data and critical revisions. Anthony Charles contributed to conception and study design, revising manuscript.

Funded by the National Institute of Health, Fogarty International Center grant D43TW009340.

\section{Disclosure}

The authors report no proprietary or commercial interest in any product mentioned or concept discussed in this article.

\section{R E F E R E N C E S}

1. World Health Organization. The global burden of disease: 2004 update; 2008. Geneva: World Health Organization; 2008. www. who.int/healthinfo/global_burden_disease/GBD_report_2004 update_full.pdf. Accessed November 2, 2019.

2. Stokes MAR, Johnson WD. Burns in the third world: an unmet need. Ann Burns Fire Disasters. 2017;30:243-246.

3. Peden MM, Oyebite K, Ozanne-Smith J, et al. World report on child injury prevention. Geneva, Switzerland: World Health Organization; 2008.

4. World Health Organization. Deaths estimates for 2008 by cause for WHO member states. Geneva, Switzerland: World Health Organization; 2011.

5. Edwards D, Heard J, Latenser BA, Quinn KY, van Bruggen J, Jovic G. Burn injuries in eastern Zambia: impact of multidisciplinary teaching teams. J Burn Care Res. 2011;32:31-38.

6. Jackson DM. The evolution of burn treatment in the last 50 years. Burns. 1991;17:329-334.

7. Wasiak J, Cleland H, Campbell F. Dressings for superficial and partial thickness burns. Cochrane Database Syst Rev. 2008:CD002106.

8. Manktelow A. Burn injury and management in Liberia. Burns. 1990;16:432-436.

9. Sowemimo GO. Burn injuries in Lagos. Burns. 1983;9:280-283. 
10. Laloe V. Epidemiol mortality burns a General Hospital of Eastern Sri Lanka. Burns. 2002;28:778-781.

11. Gallaher JR, Mjuweni S, Shah M, Cairns BA, Charles AG. Timing of early excision and grafting following burn in subSaharan Africa. Burns. 2015;41:1353-1359.

12. Munster AM, Smith-Meek M, Sharkey P. The effect of early surgical intervention on mortality and cost-effectiveness in burn care, 1978-91. Burns. 1994;20:61-64.

13. Janžekovič Z. A new concept in the early excision and immediate grafting of burns. J Trauma. 1970;10:1103-1108.

14. Burke JF, Quinby Jr WC, Bondoc CC. Primary excision and prompt grafting as routine therapy for the treatment of thermal burns in children. Surg Clin North Am. 1976;56:477-494.

15. Nguyen TT, Gilpin DA, Meyer NA, Herndon DN. Current treatment of severely burned patients. Ann Surg. 1996;223:14-25.

16. Libby P. Inflammatory mechanisms: the molecular basis of inflammation and disease. Nutr Rev. 2007;65:S140-S146.

17. Puri V, Khare NA, Chandramouli MV, Shende N, Bharadwaj S. Comparative analysis of early excision and grafting vs delayed grafting in burn patients in a developing country. J Burn Care Res. 2016;37:278-282.

18. Puri V, Khare NA, Chandramouli MV, Shende N, Bharadwaj S. Comparative analysis of early excision and grafting vs delayed grafting in burn patients in a developing country. $J$ Burn Care Res. 2016;37:278-282.

19. Ong YS, Samuel M, Song C. Meta-analysis of early excision of burns. Burns. 2006;32:145-150.
20. Gallaher JR, Mulima G, Kopp D, Shores CG, Charles AG. Consequences of centralised blood bank policies in subSaharan Africa. Lancet Glob Health. 2017;5:e131-e132.

21. Hyder A, Kashyap K, Fishman S, Wali S. Review of childhood burn injuries in Sub Saharan Africa: a forgotten public challenge. Afr Saf Promot. 2012;2:43-58.

22. Fomukong NH, Mefire AC, Beyiha G, Lawrence M, Edgar MML, Nkfusai NC. Cumber SN predictors of mortality of pediatric burn injury in the douala general hospital, Cameroon. Pan Afr Med J. 2019;33:189.

23. Outwater AH, Thobias A, Shirima PM, et al. Prehospital treatment of burns in Tanzania: a mini-meta-analysis. Int J Burns Trauma. 2018;8:68.

24. Rybarczyk MM, Schafer JM, Elm CM, et al. A systematic review of burn injuries in low-and middle-income countries: epidemiology in the WHO-defined African Region. Afr J Emerg Med. 2017;7:30-37.

25. Karan A, Amado V, Vitorino P, Kulber D, Taela A, DeUgarte DA. Evaluating the socioeconomic and cultural factors associated with pediatric burn injuries in Maputo, Mozambique. Pediatr Surg Int. 2015;31:1035-1040.

26. Onuba O, Udoidoik E. The problem and prevention of burns in developing countries. Burns. 1987;3:382-385.

27. Buchaanan RC. Cause and prevention of burns in Malawi. Cent Afr J Med. 1972;18:55-56.

28. deVries CR, Rosenberg JS. Global surgical ecosystems: a need for systems strengthening. Ann Glob Health. 2016;82:605-613. 